\title{
Frontières
}

\section{De la prévention du suicide comme une question sociologique}

\section{Matthieu Lustman}

Volume 21, numéro 1, automne 2008

Prévenir le suicide

URI : https://id.erudit.org/iderudit/037870ar

DOI : https://doi.org/10.7202/037870ar

Aller au sommaire du numéro

Éditeur(s)

Université du Québec à Montréal

ISSN

1180-3479 (imprimé)

1916-0976 (numérique)

Découvrir la revue

Citer cet article

Lustman, M. (2008). De la prévention du suicide comme une question sociologique. Frontières, 21(1), 15-22. https://doi.org/10.7202/037870ar

\section{Résumé de l'article}

Historiquement approché à travers le prisme de la morale (théologie, philosophie), le suicide relève depuis le $\mathrm{XIX}^{\mathrm{e}}$ siècle de l'analyse scientifique (psychiatrie, sociologie...). Son étude est-elle pour autant objective, exempte de croyance ? Il s'agit ici d'interroger la démarche scientifique : ne faut-il voir dans le suicide qu'un problème social à résoudre ou l'analyser comme un problème sociologique, un fait social total ? Étudier, par exemple, suicidologie et prévention du suicide dans l'ensemble de leurs dimensions, et pas uniquement dans une logique d'évaluation, permet de révéler valeurs, normes, facteurs socioculturels sous-jacents influençant théorie et pratique des soignants, d'observer comment les individus intègrent les enjeux de prévention et de sortir de l'aporie des critiques paradoxales adressées à la prévention (inefficacité / contrôle social) en montrant son rôle d'agent de transformation sociale 


\section{Résumé}

Historiquement approché à travers le prisme de la morale (théologie, philosophie), le suicide relève depuis le $\mathrm{XIX}^{\mathrm{e}}$ siècle de I'analyse scientifique (psychiatrie, sociologie...). Son étude est-elle pour autant objective, exempte de croyance? II s'agit ici d'interroger la démarche scientifique: ne faut-il voir dans le suicide qu'un problème social à résoudre ou l'analyser comme un problème sociologique, un fait social total? Étudier, par exemple, suicidologie et prévention du suicide dans l'ensemble de leurs dimensions, et pas uniquement dans une logique d'évaluation, permet de révéler valeurs, normes, facteurs socioculturels sous-jacents influençant théorie et pratique des soignants, d'observer comment les individus intègrent les enjeux de prévention et de sortir de l'aporie des critiques paradoxales adressées à la prévention (inefficacité / contrôle social) en montrant son rôle d'agent de transformation sociale.

Mots clés: suicide - prévention suicidologie - sociologie - autonomie culture

\section{Abstract}

Historically, the study of suicide was coming through the prism of morality (theology, philosophy). Since the 19th century, suicide is studied with a scientific approach (psychiatry, sociology). Is that study nevertheless objective, and free of belief? We have to question the scientific approach: should we see in suicide, a social problem to solve or should we analyze it as a sociological problem? Considering for example suicidology and suicide prevention, in all their dimensions and not only in an assessment approach, can reveal values, standards, cultural factors underlying, influencing theory and practice of health care; this can permit to observe how individuals include the questions of prevention, and break the paradoxical aporia criticism directed at prevention (inefficiency, social control) showing its role as an agent of social change.

Keywords: suicide - prevention suicidology - sociology - autonomy culture.

$\begin{array}{llllll}R & \mathrm{~T} & \mathrm{C} & \mathrm{L} & \mathrm{E} & \mathrm{S}\end{array}$

\title{
De la prévention
} du suicide comme une question sociologique

\author{
«IL FAUT RÉFLÉCHIR POUR MESURER \\ ET NON MESURER POUR RÉFLÉCHIR »
}

BACHELARD

\section{Matthieu Lustman, \\ docteur en médecine et en sociologie, maître de conférences, enseignant-chercheur, Université catholique de Lille, Centre d'éthique médicale, UNPS, Comités scientifique et éthique.}

Le suicide peut-il être étudié uniquement dans une perspective de santé publique, c'est-à-dire comme un problème social qu'il faut expliquer et résoudre? Ou ne serait-il pas plus heuristique de l'étudier comme un problème sociologique, c'est-àdire comme un fait social total au sens de Mauss? Se poser cette question revient à s'interroger sur la manière dont est étudié le suicide. Pendant des siècles, les approches philosophique et religieuse ont prévalu : la méthode consistait à chercher à établir par un raisonnement si « la mort volontaire est en soi chose licite ou illicite» (Bayet, 1922, p. 5). Entre la condamnation sans appel de saint Augustin et les interrogations d'Érasme (L'éloge de la folie), les questions de morale et de sens étaient essentielles: se donner la mort était-il un acte de lucidité, de liberté ou la pire des lâchetés, le refus du don de Dieu, avec risque de damnation éternelle? À partir du XIX ${ }^{\mathrm{e}}$ siècle s'amorce une révolution dans l'histoire du suicide: l'approche scientifique a progressivement «supplanté le débat moral» (Debout, 2002, p. 18). Peut-on désormais considérer que l'étude du suicide est devenue neutre, exempte de croyance?

L'étude scientifique du suicide privilégie une approche clinique: décrire, expliquer. Elle a permis de lever en partie le tabou (le suicide n'est plus considéré comme un crime) et de mettre en œuvre une politique de prévention (le suicide est passé du juriste au médecin). Cependant, selon les analyses philosophiques récentes (Alvarez 1972; Amery 1976; Guirlinger, 2000), cette démarche a un prix: la suicidologie aurait oblitéré la question du sens. Selon Guirlinger, il n'existerait pas de séparation radicale entre approche religieuse et scientifique mais des points de convergence: dans les deux cas, le suicide représente «une négativité absolue». La suicidologie descriptive ne se construirait-elle pas aux dépens du sens? Cette question en masque une autre: en esquivant la recherche du 
sens, n'est-ce pas la place et le rôle que le suicide occupe dans la société qui ne sont pas abordés? Autrement dit, peut-on se limiter à analyser le suicide à la manière de Durkheim (1897), comme un objet dont il faut trouver la cause sociale, ou ne faudrait-il pas l'étudier aussi comme un fait social total, c'est-à-dire dans l'ensemble de ses dimensions? Ne pas voir dans le suicide uniquement un problème social mais en faire une question sociologique?

\section{LE COUPLE COURAGE / LÂCHETÉ}

\section{S'EFFACE DEVANT L'EXPLICATION}

\section{PAR LA SOUFFRANCE,}

\section{LA MALADIE MENTALE,}

\section{LA PERTE DU LIEN SOCIAL.}

Étudier le suicide et sa prévention comme une question sociologique n'est pas uniquement affaire de théorie mais peut aussi éclairer la pratique. En nous appuyant sur Beck (approche réflexive de la science qui soumet les discours scientifiques au regard critique, 1986), sur Fassin (un problème de santé ne correspond pas à une réalité naturellement objective fondée sur l'évolution des connaissances mais résulte d'un processus complexe de négociation, Dozon et Fassin, 2001) et sur Burton-Jeangros (approche constructiviste de la réalité qui considère que la gestion des risques par le public intègre à côté de la logique médicale des paramètres sociaux et culturels, 2004), nous partirons de l'hypothèse que la prévention du suicide ne résulte pas seulement de l'évolution du savoir médical mais correspond aussi à un phénomène de construction sociale au sens de Berger et Luckmann (1966). À partir d'une approche sociologique ${ }^{1}$, nous explorerons quelques pistes de réflexion (Le suicide est-il encore un problème moral ? Quelles sont les causes de l'émergence de la prévention du suicide? La prévention est-elle identique d'un pays à l'autre? De quelle manière la prévention s'inscrit-elle dans les politiques de santé publique?) et nous étudierons la place de la prévention dans nos sociétés: existe-t-il un ou plusieurs modèles de prévention?

\section{DE NOUVEAUX ENJEUX MORAUX}

Le suicide est-il encore un problème moral? La question semble désuète. En effet, le suicide est de moins en moins condamné. "Se tuer» est perçu par une majorité de Français (80\%) comme un signe de grande souffrance, d'appel au secours. Ils ne sont que $10 \%$ à y voir un acte de lâcheté et $7 \%$ de courage (sondage UNPS, 2000). Le couple courage / lâcheté s'efface devant l'explication par la souffrance, la maladie mentale, la perte du lien social. Le suicide étant de moins en moins sujet à jugement, est-il devenu un objet d'étude scientifique neutre, dépassionné, sans enjeu? L'analyse anthropologique (Dozon et Fassin, 2001) montre que les politiques de santé publique ne relèvent pas uniquement d'une logique scientifique fondée sur une "connaissance positive » après «mise en statistique du réel», mais s'appuient aussi sur des valeurs, des normes, c'est-à-dire sur une forme de morale. Ces dernières, intériorisées sous forme d'évidences partagées, de lieux communs, sont peu questionnées par les acteurs. En analysant le dialogue entre suicide et morale, il est possible de faire émerger les valeurs sous-jacentes qui influencent les pratiques. Le suicide relèverait toujours d'enjeux moraux mais ils auraient évolués.

\section{LE CRIME N'EST PLUS}

DU CÔTÉ DU SUICIDE

De moins en moins objet d'opprobre moral, le suicide est plutôt perçu comme un signe de désespoir. Si cette dernière notion a toujours été associée au suicide, elle n'est plus interprétée de la même manière. Se donner la mort n'est plus céder à la tentation du désespoir, du diable, mais constitue un acte lié aux souffrances intenses que subit l'individu, lui interdisant tout autre choix. Dans cette logique, le raisonnement moral s'inverse progressivement. Pendant des siècles le fait de se tuer a été considéré comme un acte immoral parce que ce geste enfreignait les lois de Dieu ou de la Nature. De nos jours, ce qui est mis en avant est l'impasse, la souffrance subie. En conséquence, le regard sur le suicidé évolue: de criminel (qui pouvait susciter la pitié; Dechoz, 1994), il tend de plus en plus à être considéré comme une victime (qu'il ne faut pas juger). En revanche, ce qui ne change pas, c'est le traumatisme que cause le suicide, il est toujours perçu comme « un comportement ayant des effets dévastateurs sur le tissu cohésif de la société [... avec] comme conséquence beaucoup de douleur, de rupture dans la vie des individus, des familles et des communautés » (ONU, 1996, cité par Aujard, 2007, p. 171). Ainsi le jugement moral demeure, mais ne porte plus sur le même objet et entraîne de nouveaux devoirs :

- la nécessité de trouver des stratégies pour « réduire l'expression et les conséquences du comportement suicidaire » (ONU, 1996, cité par Aujard, p. 171). Longtemps considéré comme une fatalité (à l'instar des catastrophes naturelles), le suicide s'est mué de destin tragique en mort prématurée évitable (l'accroche de l'UNPS n'est-elle pas: "Le suicide n'est pas une fatalité»). L'obligation morale de prévenir le suicide s'impose peu à peu, doublée, comme le souligne Facy (Facy et Debout, 2006), d'une quasi-obligation pour chaque pays de présenter de bons indicateurs de santé publique;

- qui dit victime dit aussi coupable, il faut donc trouver un responsable. En conséquence, s'il ne s'agit plus de juger le cadavre comme au Moyen-Âge, s'amorce l'idée de juger la personne ou le système reconnu responsable. La jurisprudence évolue: pour la première fois, l'État a été condamné en France pour le suicide d'un détenu en prison.

\section{DES RAPPORTS ENTRE SUICIDE}

\section{ET AUTONOMIE}

Le suicide est perçu comme le signe de l'échec d'un individu, mais aussi de son entourage, de la société et de ses dirigeants: il remet en cause "les relations humaines fondamentales» (Minois, 1995). Étant considéré comme un trouble à l'ordre public, il impose, sous une forme ou une autre, un traitement social. De la condamnation à la prise en charge médicale, les modes de prise en charge ont varié, ces évolutions peuvent s'expliquer par les transformations progressives des rapports entre individu et société. Pour l'individu en société, les valeurs de référence ont longtemps été l'honneur, l'obéissance, l'esprit de sacrifice, la recherche du salut. De nos jours de nouvelles valeurs prédominent: l'autonomie, le bonheur sur terre et la sacralisation de la vie. Ces évolutions étant concomitantes avec la transformation du traitement social du suicide, il faut se demander comment ces nouvelles valeurs influencent le regard que nous portons sur le suicide. Lorsque les valeurs d'obéissance et de recherche du salut prédominaient, se donner la mort était perçu à travers le prisme moral du suicide et du sacrifice. Le premier était condamné par la société (suicide égoïste/anomique) et le second valorisé (suicide altruiste/ fataliste). Dans cette logique, le jugement moral ne portait pas tant sur l'acte que sur son intention: sacrifier sa vie (pour autrui, sa patrie, une idée) était l'acte de noblesse par excellence (Caton, Socrate), se tuer pour des raisons personnelles était le pire des crimes (Judas), la vie de l'individu ne lui appartenait pas (Dauzat, 1998). De nos jours, valorisation de l'autonomie et sacralisation de la vie transforment le rapport individu-société : les choix personnels priment la communauté. Cette évolution n'est pas sans incidence sur la manière d'appréhender le suicide: le sacrifice perd 
de sa noblesse, il est interprété comme une dépression masquée et parallèlement émerge l'idée d'un "droit au suicide» (Debout, 2002), illustré par la polémique autour du livre Suicide mode d'emploi (1982). Si l'idée d'un droit au suicide reste marginale, elle n'en influence pas moins notre manière d'aborder le problème. Une nouvelle question apparaît: dans quelle mesure est-il moralement légitime de s'opposer à la volonté de l'individu et à ce qui pourrait être considéré comme un choix? Le débat se trouve renouvelé: la question morale ne porte plus sur l'acte (se donner la mort est-il licite? Dans quel cas s'agit-il d'un acte noble, d'un crime?) mais sur les situations où il est légitime de respecter la volonté d'un individu d'être maître de son destin.

\section{SUICIDE ET EUTHANASIE}

La différence de traitement entre le suicide et l'euthanasie illustre cette situation. Actuellement, ces deux problématiques sont considérées comme des objets différents. Cette différence est-elle de nature ou est-elle sociale? Observer la manière dont est pris en charge le suicide permet de comprendre une des limites du principe d'autonomie: le principe de vie. Il justifie de ne pas respecter le choix des individus lors de tout suicide, quel qu'en soit le motif. Il n'existe qu'une seule exception : lorsque la mort approche, la question du sens peut redevenir légitime, comme l'a montré le débat en France autour de la loi Leonetti. La valorisation de l'autonomie et la sacralisation de la vie renversent les perspectives morales: la question de l'euthanasie est légitimée, celle du sacrifice dévalorisée. Cette logique sépare le suicide de l'euthanasie et structure les modes d'approche: le suicide relève presque exclusivement de la science et de la prévention, l'euthanasie davantage de questions philosophiques, morales ou d'ordre judiciaire. Le débat entre suicide et morale semble loin d'être clos: il se renouvelle en fonction des enjeux d'une époque donnée et influence les pratiques. Reposant sur une approche scientifique, la prévention du suicide s'appuie aussi sur des normes et des valeurs (autonomie, sacralisation de la vie, diminution obligatoire des morts évitables...) dont l'étude est nécessaire pour comprendre les enjeux, les réussites, les échecs de celle-ci ainsi que ses bases théoriques.

\section{ÉMERGENCE DE LA PRÉVENTION DU SUICIDE}

\section{UN PROBLÈME DE DÉFINITION}

Existe-t-il une ou plusieurs manières de concevoir la prévention du suicide? La plupart du temps, cette question n'est pas posée. Batt (Batt-Moillo et Jourdain, 2005, p. 183-185), en présentant les différents modèles de prévention, montre pourtant toute la richesse de cette interrogation. Avant d'aborder ces modèles, nous avons tout d'abord essayé de savoir s'il existait, pour les acteurs, une ou plusieurs définitions de la prévention. Nous avons interviewé une vingtaine d'intervenants et de chercheurs en suicidologie. La prévention du suicide peut être définie comme «l'ensemble des activités théoriques et pratiques dont le but est de prévenir le passage suicidaire et ainsi de diminuer le nombre de morts par suicide», ou comme «l'ensemble des mesures mises en œuvre en vue d'éviter le passage à l'acte suicidaire (formation au repérage, à la communication avec les patients, à la sécurisation des lieux, etc.) ». Dans ces définitions se trouve une logique sanitaire, où "prévention du suicide» se résume à «éviter le passage à l'acte». D'autres définitions proposent une approche plus large: "l'ensemble des actions entreprises dans le champ social, psychologique, biologique, au sein d'une population, en vue d'une réduction de l'incidence du suicide». Il s'agit toujours de diminuer les taux, mais l'enjeu devient sociétal: le suicide est inscrit dans l'ensemble de ses dimensions. Enfin, la prévention peut aussi être définie dans une vision globale comme "un état d'esprit, une mise en place de dispositifs, de réflexion, d'outils, de concertation pour proposer une qualité de vie, des attitudes, des lieux d'écoute et de soins, en vue d'élargir le champ des possibles autres que la mort». Elle repose alors sur l'idée que le suicide n'existe pas uniquement en lui-même, mais qu'il faut aussi tenir compte de ce qu'il révèle.

Derrière ces définitions se cachent des conceptions différentes: limiter le suicide à la pathologie mentale ou insister sur ses dimensions sociales avec des choix très concrets. Faut-il plutôt prioriser une intervention intensive lors des risques de passages à l'acte ou augmenter les ressources dans lesquelles l'individu pourra puiser au moment de la crise? (Aujard, 2007.) Plus précisément, il existerait deux modèles théoriques de prévention: maladie/santé (Tudor, 1996) et d'interaction ou de crise (Hawton et Van Heeringen, 2000; BattMoillo, 2001). Dans le premier, le suicide est considéré comme une pathologie, la démarche est médicale, centrée sur l'individu (repérer les personnes à risques et les prendre en charge en s'appuyant sur de bonnes pratiques). Dans le second, le suicide résulte d'une crise bio-psychosociale, aucun facteur n'est prédominant. Il y a idéation suicidaire sous l'effet de stress psychosociaux et passage à l'acte lorsqu'un évènement de vie particulière- ment déstabilisant entraîne l'individu dans une impasse. La prévention est autant centrée sur l'individu que sur la population en général, et relève alors d'une logique de santé publique. Dans l'opposition de ces deux modèles, nous retrouvons notre débat initial: le suicide comme un problème à résoudre, la médecine étant l'outil adéquat versus le suicide comme une souffrance psychologique qui peut faire sens (symptôme révélateur d'un malaise sociétal) associant les approches sociologique et psychiatrique. Or, ces modèles ne conduisent pas au même choix de prévention primaire, secondaire ou tertiaire (Batt-Moillo et Jourdain, 2005, p. 184). Cela conduit à deux questions: pourquoi tel modèle émerge à un moment donné et quelles en sont les implications pratiques sur le terrain? Le choix de privilégier tel ou tel modèle semble lié à une évolution des théories plutôt qu'à la démonstration qu'un des deux modèles est plus «performant». Si les valeurs transparaissent dans les différents modèles théoriques, il convient de voir si ce sont les seuls facteurs qui influencent la prévention ou s'il n'existe pas aussi des facteurs d'ordre socioculturel.

\section{LA PRÉVENTION DU SUICIDE}

Si la question du suicide paraît aussi ancienne que l'homme lui-même, celle de la prévention est récente (Debout, 2002, p. 30). L'analyse socio-historique de la prévention depuis les années 1950 montre que ni une augmentation radicale des occurrences ni une crise majeure du lien social ne peuvent expliquer cette évolution. En fait, deux logiques sont à l'œuvre: une logique interne liée à la mobilisation des acteurs du champ du suicide (associatif, endeuillé, épidémiologiste, psychiatre), une externe liée à l'évolution de la société (médecine, économie, évaluation, victimologie). Une description socio-historique de la prévention du suicide en France fait apparaître trois grandes étapes: les prémices de 1950 à 1970, une phase de développement de 1969 à 1993, une phase de reconnaissance institutionnelle et de premières évaluations depuis 1994.

Avant 1950, la question de la prévention ne se posait qu'en cas de pathologie mentale sévère. Il existait une séparation nette entre suicide et tentative de suicide: le premier était l'aboutissement fatal d'un trouble psychiatrique et la seconde était jugée non sérieuse. Entre un risque gravissime mais rare et des tentatives sousestimées, il y avait peu de place pour la prévention. La question de la prévention a émergé sous l'impulsion de plusieurs acteurs comme Chad Varah, Ringel, Stengel et Shneidman. Chad Varah est considéré 
comme l'inventeur de la téléphonie sociale. Avec la création à Londres des Samaritains (1953), il inscrit la prévention du suicide dans le champ du bénévolat. Ringel et Stengel ont souligné l'importance de la prise en charge médicale de toutes les tentatives de suicide même hors contexte psychiatrique grave. Ringel crée à Vienne le premier Centre de prévention du suicide (CPS) et propose un modèle qui sera repris dans de nombreux pays. Shneidman participe à la création du CPS de Los Angeles et propose le terme de suicidologie (1968); il contribue aussi à la reconnaissance du rôle des endeuillés. La prévention s'est d'abord organisée à côté du monde médical, définissant un paysage institutionnel fondé sur l'alliance des écoutants, des endeuillés et de certains psychiatres/psychologues isolés. Cette étape se traduit en France par la création d'association comme SOS Amitiés (1961), inspirée par les Samaritains. La deuxième période s'ouvre par l'accréditation par l'OMS en 1960 de l'International Association for Suicide Prévention (IASP) dont l'objectif est «d'empêcher le comportement suicidaire, à en alléger ses effets, et à fournir un forum pour des universitaires, des professionnels de la santé mentale...»(Aujard, 2007, p. 169). IASP en partenariat avec l'OMS et l'ONU va participer à l'institutionnalisation progressive de la prévention du suicide soit par le biais de conférences internationales, soit par la production d'un premier document en 1969 qui «invite les pays membres à changer les pratiques et les connaissances sur le suicide»(Aujard, 2007, p. 169). En France, la création du GEPS, organisation de psychiatres, participe de cette même logique et complète en 1969 la démarche des associations. Les recherches en épidémiologie (Davidson et Philippe, 1986) et les analyses sur le coût économique du suicide (étude RCB en 1971) se développent. En parallèle avec la création des services d'urgence, certains psychiatres (Vedrinne à Lyon, Soubrier à Paris) proposent des prises en charge particulières des tentatives de suicide (TS). En 1992, Pommereau crée la première unité spécialisée de prise en charge des jeunes suicidants. En même temps, les associations se multiplient et se spécifient (Vivre son deuil, SOS Phénix Suicide...). Ces initiatives légitiment progressivement la prévention, mais restent isolées et ne sont pas encore systématisées. La troisième période s'ouvre avec le rapport au Conseil économique et social de Debout en 1993. En 1994, le suicide est reconnu par le HCSP comme priorité de santé publique. Cela entraîne le développement de PRS (Plans régionaux de santé) dans 11 régions. En 1996 a lieu la première JNPS (Journée nationale de prévention du suicide) et a pour conséquence en 1998 la naissance de l'UNPS (Union d'associations du champ de la prévention). En 2000 a lieu la conférence de consensus et la première stratégie nationale se met en place. À partir de 2005 ont lieu les premières évaluations. En parallèle, les protocoles de prise en charge des TS se systématisent dans tous les hôpitaux et les associations continuent à se développer.

L'analyse sociologique de cette évolution montre l'émergence progressive d'un réseau stable et organisé, "la prévention du suicide mobilise tout un réseau formellement agencé en un système technico-institutionnalisé» (Aujard, 2007, p. 172). Ainsi, progressivement, la prévention du suicide devient une "réalité en marche et s'implante dans les couches de la vie sociale et quotidienne des gens» (Aujard, p. 172). Au-delà de la prévention d'un risque particulier, il s'agit dans l'idéal de la prévention primaire, secondaire et tertiaire, d'aider à la fois les individus à gérer mieux leur vie tout en leur offrant ressources et soutien lors des crises. L'analyse de cette évolution montre aussi que la prévention ne s'impose pas comme une évidence en fonction de besoins qui seraient identifiés. Bien au contraire, il s'agit d'un processus complexe où interviennent de multiples acteurs relevant de plusieurs champs d'expertise, spécialisés dans le domaine du suicide ou non. Une combinaison de facteurs directement ou indirectement liés au champ du suicide explique l'émergence de la prévention. Les facteurs directs les plus significatifs sont la conséquence des actions menées par: les associations (la téléphonie sociale a fait remonter des souffrances invisibles), les endeuillés (l'obligation progressive de tenir compte du deuil après suicide), les psychiatres (prise en compte de toutes les tentatives de suicide), les épidémiologues (possibilité de mesurer une réalité peu connue), les CPS (possibilité d'organiser la prévention et de faire travailler en coopération l'ensemble des acteurs) ou encore les institutions (la légitimation de la problématique permet de systématiser la prise en charge). Les facteurs indirects sont aussi nombreux: le développement des services d'urgence et les techniques de réanimation ont multiplié les possibilités de prise en charge des TS, le rapprochement de l'hôpital psychiatrique et de l'hôpital général a permis l'arrivée des psychiatres aux urgences, le processus de psychologisation qui prend en charge de nouvelles souffrances, l'épidémiologie qui développe la notion de «mort prématurée évitable», la rationalisation des soins dans une logique économique et la coordination des soins et, enfin, le rôle joué par le développement de la victimologie (Erner, 2006).
Les facteurs qui influencent la prévention sont multiples, confirmant le fait que l'émergence de la prévention correspond à une logique de construction sociale issue de négociations complexes. En conséquence, les enjeux ne sont pas limités à diminuer l'incidence du suicide mais, sous couvert de prévention, répondent à de multiples objectifs établis par des acteurs aux motivations variées: diminuer le tabou, gérer le drame du suicide dans les familles, prendre en charge des nouvelles formes de souffrance psychologique, aider les soignants à traiter un problème qui les met en échec, proposer de bons indicateurs dans les comparaisons internationales, baisser les coûts, rationaliser l'organisation. Il faut noter l'absence des suicidants parmi les acteurs qui influencent l'organisation de la prévention. Notre analyse est pour l'instant restée théorique, pour étudier la prévention comme un phénomène social total, il faut maintenant plonger au cœur des pratiques.

\section{ESQUISSE D'UNE COMPARAISON DES MODES DE PRÉVENTION, ENJEU D'ÉVALUATION}

Comme la prévention du suicide résulte d'un processus de construction sociale, elle ne s'est probablement pas développée de manière identique dans chaque pays. En nous appuyant sur la revue de la littérature, nous comparerons quelques stratégies nationales, puis sur une comparaison de l'éthique des soignants, de la téléphonie sociale ${ }^{2}$ et des formations à la crise suicidaire, nous analyserons les différences de pratiques et observerons les facteurs qui les influencent.

\section{LES STRATÉGIES NATIONALES}

Pour comparer les logiques de prévention, la démarche habituelle est de présenter soit les différentes stratégies nationales (Batt-Moillo et Jourdain, 2005), soit des méta-analyses (Silverman, 2005; Diego de Leo, 2005). Selon Diego de Leo, les stratégies se ressemblent sur de nombreux points (mieux repérer et traiter les troubles mentaux, diminuer l'accès aux moyens létaux, améliorer l'accès aux services de santé mentale), mais diffèrent pour certains objectifs (ciblage général ou de populations à risques, destinées aux jeunes ou aux personnes âgées, prioriser uniquement la logique médicale ou associer des objectifs sociétaux: qualité de vie, chômage, exclusion sociale...), axer la recherche sur l'épidémiologie ou sur une évaluation plus générale, prendre en compte ou non les enjeux d'organisation. Ce type d'analyse permet d'esquisser des comparaisons internationales, mais demeure limitée. En effet, les comparaisons effectuées ne 
portent que sur les objectifs affichés, sans observation des pratiques et sans tenir compte des bilans d'évaluation. Comparer les objectifs affichés permet cependant de souligner trois éléments: les choix théoriques influencent réellement les pratiques (privilégier une approche médicale ou sociétale génère des stratégies différentes); des divergences apparaissent entre logique théorique et pratique (en théorie, le modèle bio-psycho-social semble avoir la cote auprès des chercheurs, en pratique, lorsqu'il faut sélectionner des objectifs concrets, le modèle médical semble privilégié) ; les valeurs et les facteurs socioculturels transparaissent à travers les objectifs (s'adresser aux jeunes plutôt qu'aux personnes âgées, se focaliser sur les groupes à risques ou la population générale, accorder du sens au suicide ou non, se référer à telle ou telle discipline scientifique...) et amènent à privilégier certains axes. Ainsi il existe une première stratégie orientée vers la résolution du problème dans le seul but de diminuer les suicides (l'objectif est clair) et une seconde, qui intègre de manière complexe et disparate l'idée que le suicide est aussi un fait de société (le but est plus flou).

\section{LES PRATIQUES DE TERRAIN}

Comparer les différents modes de prévention implique d'étudier les pratiques dans chaque pays, au-delà des théories. À ce sujet, il est déjà possible de donner quelques éclairages à partir de comparaisons entre l'éthique, la téléphonie sociale et les formations à la prévention du suicide.

Pour les soignants, en théorie, le principe de vie fait consensus. Mishara (Chagnon et Mishara, 2004) a montré que, sur le terrain, les enjeux sont, dans la pratique, plus complexes. Ainsi, les acteurs de terrain sont prisonniers d'au moins deux impératifs éthiques opposés : le respect de l'autonomie et la sacralisation de la vie. D'un soignant à l'autre varie le fait de savoir jusqu'où il est légitime d'intervenir : ne pas respecter la volonté d'une personne dans le but de protéger sa vie. En fonction de ses propres valeurs, le soignant est dans l'obligation de trouver un compromis fragile. Pour Mishara, il existe des soignants interventionnistes (aucune liberté n'est possible), libertaires (la liberté fait partie de l'équation) et relativistes (tenir compte de la liberté dans certaines situations). La prise de décision repose alors autant sur un savoir que sur une éthique.

La téléphonie sociale a fait l'objet de nombreuses recherches. Aucune ne permet de mesurer de manière scientifique ses effets sur les taux de suicide (Hardy, 2000; Mishara et al., 2005), ni de montrer avec certitude qu'un type d'écoute est plus per- formant. Au Québec, la téléphonie sociale est clairement structurée avec deux types de ligne: d'intervention ou d'écoute. Les premières sont composées de bénévoles et de professionnels, l'écoute est plutôt directive, orientée vers la résolution des problèmes et se concentre uniquement sur le suicide, les appels relevant d'une souffrance générale sont orientés vers les secondes lignes qui proposent une écoute non directive de type rodgerien. En cas de passage à l'acte, l'intervention médicale est systématique. En France, il existe de multiples lignes soit bénévoles, soit professionnelles. Elles peuvent être centrées sur la souffrance en général, le suicide ou un problème particulier (maladie, âge...). Qu'elles soient générales ou spécifiques, elles ne sélectionnent pas les appels et proposent un type d'écoute « considéré» comme peu directif (rodgerien, counseling...). En cas de passage à l'acte, pour un certain nombre d'associations, l'intervention nécessite l'accord de l'appelant. Si l'objectif est le même (diminuer les taux de suicide), au Québec, une démarche structurée et directive est privilégiée, centrée sur le suicide et utilisant de nombreux référentiels théoriques. En France, la démarche est plus indirecte, davantage centrée sur la souffrance en général avec l'idée qu'une écoute empathique permet à la personne de trouver elle-même les ressources nécessaires pour faire ses propres choix. Nous retrouvons donc la tension entre les deux modèles: au Québec, la téléphonie sociale semble davantage centrée sur le suicide comme problème à résoudre. L'individu est encadré, l'autonomie semble secondaire. En France, la prévention primaire est mise en avant, la question du sens est prédominante. L'individu est soutenu tout en étant renvoyé à son autonomie.

Les formations occupent une place centrale dans les stratégies nationales. Si les objectifs sont identiques, les pratiques diffèrent. En France, les formations TerraSeguin se passent le plus souvent à l'hôpital. Elles s'adressent à tout professionnel du champ social et sanitaire demandeur. L'objectif est triple: mieux repérer en posant directement la question du suicide, évaluer le niveau de risque et former des personnesressources dans le but d'initier des réseaux. En Belgique, le Centre de prévention de Bruxelles propose une autre démarche: les formations se font souvent dans les institutions où les problèmes se posent, il existe un temps d'évaluation de chaque situation par l'équipe et d'adaptation de la formation. Le suicide n'est plus uniquement un problème à prévenir mais l'occasion de soulever la chape de plomb que la violence d'un suicide avait révélée. Entre la France et Bruxelles, la logique de formation diffère: le suicide est soit uniquement un problème à résoudre, soit un symptôme révélateur. Est-ce un hasard si les bilans des formations françaises induisent peu de questions, alors que les formations bruxelloises sont complexes et soulèvent davantage d'interrogations chez leurs organisateurs?

L'analyse des stratégies nationales et l'observation des pratiques permettent de voir comment se traduisent, sur le terrain, la tension entre modèle médical et bio-psychosocial ainsi que les différentes manières de gérer la tension entre respect de l'autonomie et risque de non-assistance à personne en danger. De nouveaux facteurs qui influencent la prévention apparaissent: la manière dont les intervenants gèrent les situations et dont les populations réagissent, les enjeux de gestion de l'autonomie, les enjeux culturels avec le ciblage des groupes à risques, les enjeux politiques, les modes d'évaluation des stratégies, etc. L'analyse de ces facteurs fait émerger trois types de prévention: une première centrée sur les objectifs médicaux, une deuxième qui y associe des objectifs sociétaux et une troisième qui ajouterait un regard réflexif sur la prévention en observant la méthode. Vouloir étudier la prévention comme un fait social total peut prendre sens en permettant une évaluation non pas selon «l'efficacité» mais plutôt, comme le défendent Mishara et Chagnon, selon «les processus » avec un regard critique sur la construction des méthodes. Reste alors la nécessité de recontextualiser la prévention du suicide à l'aune des politiques de santé publique, afin de poser la question du rôle de la prévention au sein de la société.

\section{DE LA PRÉVENTION DU SUICIDE ET DES POLITIQUES \\ DE SANTÉ PUBLIQUE}

Nous avons insisté sur l'importance de saisir les valeurs sous-jacentes de la prévention. En effet sans analyser ce que Fassin (Dozon et Fassin, 2001, p. 8) appelle «les implicites de la santé publique, ce qui va de soi dans le travail quotidien et qui n'est jamais expliqué", c'est-à-dire les valeurs et les facteurs socioculturels qui influencent théories et pratiques, il est difficile de comprendre non seulement la manière dont s'élaborent les politiques de prévention mais aussi, au-delà des objectifs affichés, leur rôle. Pour atteindre ce but, il est impossible de se limiter à l'étude de la prévention, comme si elle existait ex nihilo: il faut étudier la manière dont la prévention du suicide s'inscrit dans les politiques de santé publique. Un second rappel historique permet de saisir les logiques de l'émergence de la prévention puis de comprendre que la santé publique est aussi une culture, avant de conclure sur le rôle joué par la prévention du suicide. 
ÉMERGENCE DE LA SANTÉ PUBLIQUE

Si les politiques de santé publique via l'hygiénisme ne sont pas récentes, leur place actuelle est sans précédent: «la santé publique s'est imposée, dans les sociétés contemporaines, comme un lieu central de l'espace social et politique» (Dozon et Fassin, 2001, p. 7). Le sida, la vache folle, les OGM ou la lutte contre le tabac sont autant d'exemples qui illustrent cette situation. La place centrale prise par la santé publique a amené Foucault à parler de «bio-pouvoir». Selon cet auteur, la santé publique prend son envol au XVIII ${ }^{\mathrm{e}}$ siècle, avec pour la première fois la mise en place de systèmes organisés comme la nouvelle Société royale de médecine. Ces structures permanentes permettent d'engager des projets à long terme. Parallèlement à cette évolution se dessine un courant néo-hippocratique qui défend l'idée qu'une "maladie naît dans des conditions d'environnement précises, il suffit d'en changer l'un des éléments pour la supprimer» (Bourdelais, 2001, p. 11). L'idée d'améliorer la santé publique émerge avec la prise de conscience qu'il y aura des résistances, les individus ne prenant pas naturellement conscience de leur bien : il faut faire évoluer les comportements et prendre des mesures interventionnistes. Ce raisonnement rejoint celui des philosophes: Rousseau considère que «l'homme, naturellement sain, crée des déséquilibres par ses comportements, si bien que la nature se défend en développant des maladies. La médecine tente pour l'essentiel de rétablir les lois de la nature lorsqu'elles ont été transgressées, mais rencontre là ses limites. Aussi les médecins doivent-ils convaincre les princes, le pouvoir politique, afin que les lois médicales et sanitaires évitent la plupart ou les plus graves des transgressions ». Les politiques de santé publique émergent à partir d'une double bataille: convaincre les princes de se mobiliser et les populations de changer leur comportement.

Parallèlement, le $\mathrm{XIX}^{\mathrm{e}}$ siècle est aussi l'époque de quatre évolutions fondamentales: le paradis passe des cieux sur terre (les médecins deviennent les nouveaux prêtres d'une religion du bonheur terrestre); une méthode d'étude des populations apparaît: les statistiques ; l'État prend conscience que la santé des populations est une richesse et devient garant de la santé publique; avec le développement de la démocratie et du principe d'autonomie, l'État perd progressivement son pouvoir de mort sur les individus. Pour Foucault ces évolutions changent la nature du pouvoir exercé par l'État sur les individus: du droit de faire mourir, il devient un droit de faire vivre. Avec la médecine et les statistiques, l'État a les moyens de ses ambitions. Campeon (2005) fait de cette hypothèse l'idée essentielle de son analyse de l'émergence de la prévention du suicide. Elle prendrait racine dans une évolution fondamentale de la manière dont l'État conçoit son rôle et modifie sa gestion de la population: un pouvoir de faire vivre. Entre valeurs et conditions de naissance de la santé publique, nous pouvons mieux saisir l'ensemble des facteurs qui président à l'émergence de la prévention du suicide: des concepts (hygiénisme, population), des acteurs (État, chercheurs, médecins, financeurs, familles), des sciences (biologie, psychiatrie), des enjeux politiques (investissement du corps vivant ou «biopouvoir»), une méthode (les statistiques), des valeurs (sacralisation de la vie) et une philosophie (les comportements humains doivent être modifiés). Nous retrouvons une logique de construction sociale où la prévention du suicide se construit par tâtonnements et négociations plus ou moins masquées, entre des injonctions contradictoires et des champs concurrents, tout en s'adaptant à chaque étape aux enjeux de société. Le processus d'ensemble est peu perceptible et doit être décrypté; le rendre visible permet d'analyser l'ensemble des enjeux de la prévention qui s'avèrent plus complexes que prévus.

\section{LA PRÉVENTION EST AUSSI UNE CULTURE}

Deux critiques opposées sont adressées aux politiques de santé publique: soit elles ne sont pas efficaces et difficilement évaluables, soit, trop contraignantes, elles font courir le risque de contrôle social. Ainsi, la prévention oscillerait d'un monde de cauchemar à un autre: d'un côté, une « utopie vaine», de l'autre, «une forme de totalitarisme» où tout serait subordonné à un seul but: la préservation de la vie, au risque de sacrifier l'autonomie (Dozon et Fassin, 2001, p. 50-52). Ce débat caricatural est le reflet de nos désillusions ou de nos peurs. Dans cette logique, la prévention ne prendrait sens que dans ses objectifs affichés. Son impact ne pourrait être mesuré qu'à l'aune de son efficacité ou du danger qu'elle ferait courir. En fait, la réalité est plus complexe et la réflexion doit porter sur l'ensemble des conséquences d'une politique de prévention et non se contenter d'un seul type d'indicateur. L'analyse montre en effet que si la santé publique semble relever d'une logique monolithique où tous les intervenants partagent un même objectif (la diminution du nombre de suicide), dans les faits, il existe des logiques et sousobjectifs différents. Conception et objectif diffèrent entre épidémiologiste, sociologue, psychiatre, médecin généraliste, écoutant, endeuillé... Si les logiques ne sont pas les mêmes entre chaque catégorie d'acteurs, les logiques de prévention peuvent aussi varier pour un même groupe d'acteurs. En effet la prévention peut se définir selon quatre schèmes (Dozon et Fassin, 2001, p. 29-30) : le modèle magico-religieux (qui relève du sacré avec interdits et obligations), le modèle profane (qui relève de l'espace politique avec contrôle social, la forme paradigmatique en étant le cordon sanitaire), le modèle pastorien (qui relève de la science et s'inscrit sur le terrain de l'efficacité), le modèle contractuel (qui relève d'une logique de négociation avec le patient dans un but d'accord partenarial ou d'éducation à la santé). Historiquement datés, ses schèmes ne s'opposent pas mais se sédimentent, ainsi, nous retrouvons dans la prévention du suicide: une part de sacré (la vie est sacralisée), une part d'intervention coercitive (les suicidants sont «sauvés » quelle que soit leur volonté), une part scientifique (l'épidémiologie, la psychiatrie) et une part contractuelle (l'alliance thérapeutique). En outre, ces modèles se complètent plus qu'ils ne se contredisent dans une logique de sédimentation où s'entremêlent croyance, pouvoir, savoir et recherche d'efficacité.

Pour compléter l'analyse, un dernier détour s'impose: le débat entre science et culture. Selon Fassin, la santé publique n'est neutre ni politiquement, ni scientifiquement. L'épidémiologie est pourtant associée à l'image de la technique, cela présente un double avantage pour les décideurs: objectivité scientifique et opérationnalité. L'épidémiologiste serait l'expert, le «savant éclairant le politique» (Dozon et Fassin, 2001, p. 8). Cette vision des choses place la santé publique du côté de la science et le profane dont il faut modifier le comportement, du côté de la culture. L'épidémiologie permet la description du réel via les statistiques; elle mesure l'importance du problème, cible les populations à risques et donne l'impression de fonder les décisions de manière objective. Pure technique, l'épidémiologie n'énoncerait que des faits. Ce raisonnement cependant ne prend pas en compte quatre facteurs: la part de croyance qui subsiste dans l'élaboration des facteurs de risque (l'identification d'un facteur de risque ne correspond pas simplement à une mise en statistique du réel, mais se retrouve en interaction permanente avec le social: sélectionner une catégorie plutôt qu'une autre) ; la part d'idéologie (les individus sont rendus responsables de leur santé et encouragés à maîtriser leur santé en connaissance de cause par une information adéquate; Burton Jeangros, 2004); la part d'incertitude (il ne s'agit souvent que de corrélation qui n'élimine pas les facteurs intercurrents); la description statistique structure, voire invente le réel tout en le 
décrivant: elle façonne un certain type de regard et favorise des objectifs au détriment d'autres (diminuer l'incidence du suicide aux dépens de la recherche du sens). L'épidémiologie n'est donc pas qu'une technique (les statistiques): "elle est aussi une culture» (Dozon et Fassin, 2001).

Logique d'acteurs, schèmes, part culturelle de l'épidémiologie obligent à abandonner l'image d'une prévention opposant science et culture. Il y aurait illusion à croire que les facteurs socioculturels ne seraient que du côté des profanes; il existe aussi une part de croyance, de valeur, d'enjeu de pouvoir au sein des politiques de santé publique. Autrement dit, rendre visible la part de culture de la santé publique permet de relativiser la distance qui sépare professionnel et profane. Il n'y aurait pas d'un côté «un savoir positif» et rationnel et de l'autre, des profanes dont la culture serait le symbole de l'ignorance (science et culture s'opposant dans une logique du bien et du mal, du vrai et du faux). Il s'agit de prendre conscience de cette dimension sans tomber dans une dérive culturaliste avec négation de la démarche scientifique et de saisir que, si la prévention est aussi une culture, il existe alors, selon l'expression de Fassin, une «interaction entre des codes culturels ». En conséquence, la prévention, à travers les interactions entre profane et expert qu'elle induit, transforme, au nom d'un idéal "objectif », la réalité dans un sens innovant qu'aucun acteur n'aurait su prédire. Nous pouvons alors faire l'hypothèse que la prévention participe de l'évolution de la société par ses effets induits.

prévention du suicide, recherche-action élevant cette dernière au rang de "science" alors qu'elle n'est qu'un artefact publicitaire qui sert de paravent à l'expansion d'un système technique et comportemental de prévention dont l'organisation, la suicidologie, est à la fois le producteur, le développeur et le bénéficiaire» (Aujard, 2007, p. 173). Si observer la prévention du suicide à travers le risque de contrôle social permet de dévoiler la complexité des processus à l'œuvre, la radicalité de la critique doit être atténuée: la relative stabilité des taux de suicide, la faiblesse des avancées concrètes (Diego de Leo, 2005) et l'absence d'influence de la prévention sur de nombreuses pratiques sociales (peu d'influence, par exemple, sur les conditions de vie en prison ou sur le monde du travail) infirment en partie l'hypothèse. La seconde critique est l'absence de diminution significative du suicide, cet état de fait peut être relativisé par les nombreux apports de la prévention: levée du tabou, place faite aux endeuillés, modification des pratiques des soignants, reconnaissance de souffrances oubliées, modification des comportements avec une logique de psychologisation, «l'individu se verrait invité à un véritable travail sur Soi » (Macquet et Vrancken, 2006). Toute évaluation dépend du choix des indicateurs; l'approche socioanthropologique permet de montrer la complexité des actions menées. Le risque de contrôle social, par exemple peut être regardé autrement. Si, comme l'avait prédit Foucault, la santé publique a pris une place importante, il s'agit davantage

ÉTUDIER LES DÉFINITIONS DE LA PRÉVENTION MONTRE QU'IL N'EXISTE PAS DE CONSENSUS ENTRE LES INTERVENANTS: LE SUICIDE PEUT ÊTRE VU COMME UN PROBLÈME À RÉSOUDRE OU COMME UN ACTE PORTEUR DE SENS QUI DOIT ÊTRE PRIS EN COMPTE D'UN POINT DE VUE PSYCHOLOGIQUE MAIS AUSSI SOCIÉTAL.

\section{PLACE ET RÔLE}

\section{DE LA PRÉVENTION DU SUICIDE}

Les mêmes critiques sont adressées à la prévention du suicide qu'aux politiques de santé publique (contrôle social/inefficacité). En utilisant l'analyse ci-dessus, il est possible de faire une autre lecture du bilan de la prévention. La première critique est celle du risque de contrôle social, prévention du suicide et suicidologie n'auraient alors que l'apparence de la science: «en ce sens, la suicidologie est un système ouvert de recherche-action en conditions de logement, dans une logique de justice sociale, la question redevenait formulable lorsqu'il existait des enjeux de santé. Le sanitaire relégitime le social. Le concept de bio-légitimité s'adapte parfaitement à la prévention du suicide: dans le monde du travail, la logique économique prédomine. S'adapter à la mondialisation est le seul credo; il ne peut être remis en cause que lorsque la question du stress ou du suicide se pose. La prévention par ses maigres moyens et le caractère colossal de la tâche à accomplir ne peut échapper aux critiques classiques qu'en rendant visibles les enjeux socioculturels qu'elle véhicule. La prévention du suicide joue un rôle d'aiguillon qui peut apparaître modeste, mais met en valeur son rôle d'agent de transformation sociale.

$$
* * *
$$

Parti d'une interrogation sur la manière d'étudier le suicide, nous avons essayé de montrer l'intérêt d'aborder le suicide et sa prévention non comme un problème social à résoudre, mais comme un problème sociologique. S'interroger sur les rapports entre suicide et morale permet de faire apparaître les valeurs sous-jacentes. Étudier les définitions de la prévention révèle qu'il n'existe pas de consensus entre les intervenants: le suicide peut être vu comme un problème à résoudre ou comme un acte porteur de sens qui doit être pris en compte d'un point de vue psychologique mais aussi sociétal. Analyser l'émergence de la prévention nous apprend qu'elle ne repose pas uniquement sur l'évolution du savoir scientifique, mais sur une logique de construction sociale avec de nombreux enjeux. Comparer les modes de prévention fait apparaître, qu'à travers des logiques relativement proches, les choix des stratégies nationales relèvent aussi d'un contexte culturel et social, les pratiques variant d'un pays à l'autre en fonction des habitudes culturelles et des enjeux d'organisation. Enfin, en analysant la prévention du suicide à l'aune des politiques de santé publique, il apparaît qu'elle ne correspond pas uniquement à un positionnement objectif et neutre opposant de manière caricaturale des experts s'appuyant sur une logique scientifique à une population profane résistant aux bonnes pratiques pour des raisons culturelles. La prévention suppose des choix qui ne sont pas tous fondés scientifiquement, mais s'inscrit dans un contexte socioculturel. En conséquence, entre l'expert et le profane, il existe une opposition entre deux codes culturels: si l'un est davantage fondé sur un savoir scientifique, l'autre a aussi sa logique qu'il convient de prendre en compte, sans quoi il existe un risque de 
tomber dans les critiques classiques (utopie vaine/totalitarisme). Pour éviter ce piège et analyser le rôle et la place de la prévention $\mathrm{du}$ suicide, il faut rendre visibles les facteurs socioculturels qui la construisent. Deux pistes de travail apparaissent alors : 1) ne pas chercher uniquement à mesurer l'efficacité de la prévention mais étudier l'ensemble des impacts de la prévention; 2) le concept de bio-légitimité, plus adapté que le concept de bio-pouvoir, permet de montrer le rôle d'alarme sociale que joue la prévention du suicide.

La prévention du suicide se retrouve ainsi au cœur des contradictions de notre société, prise entre concurrence effrénée (principe d'autonomie) et exigence de plus en plus importante de sécurité (principe de lien social). Cette sécurité est, de nos jours, assurée en partie par le développement des politiques de santé publique. Ainsi, au concept de bio-légitimité il faut associer le concept de bio-responsabilité (Fassin). L'impératif actuel n'est plus uniquement de savoir s'alarmer, "le suicide ne doit plus être une fatalité»(UNPS) : il existe un nouveau devoir de prévenir le suicide. Le développement des politiques de prévention et un début de judiciarisation du suicide constituent les témoignages de la mise en œuvre de la bio-responsabilité dans le domaine de la mort volontaire. Cependant, l'image de la prévention ne correspond pas à la réalité observée. Entre une théorie fondée sur des savoirs scientifiquement validés qui permettent un discours normatif et prescriptif et une réalité qui inclut des facteurs socioculturels et doit s'adapter à de nombreux enjeux (économique, politique, autonomie des acteurs...), la prévention du suicide correspond, selon l'expression de Fassin, à "une discipline incertaine» complexe et imprévisible. Paradoxe d'un discours clair et net et d'une pratique qui se fonde sur le bricolage, il convient de voir dans la prévention une politique fondée sur un savoir scientifique mais aussi «un agent» parmi d'autres, qui participe à la transformation du réel. En conclusion, nous invitons à développer une recherche ayant pour but de mieux comprendre l'impact de la prévention du suicide dans chaque pays, tant sur les théories que sur les pratiques, dans une logique dont Mishara et Chagnon ont montré toute la richesse et le caractère novateur: une évaluation selon les processus et non selon l'efficacité.

\section{Bibliographie}

AMERY, J. (1996). Porter la main sur soi. Traité du suicide, Paris, Actes Sud.

ALVAREZ, A. (1972). Le dieu sauvage. Essai sur le suicide, Paris, Mercure de France.

AUJARD, M.-F. (2007). «La suicidologie, un outil de gestion du comportement", Recherches sociographiques, vol. XLVIII, $\mathrm{n}^{\mathrm{0}}$ 3, p. 161-174.

BATT-MOILLO, A. (2001). "Les facteurs de risque précédant la crise suicidaire : le point de vue du chercheur", dans FFP, La crise suicidaire, reconnaître et prendre en charge, Paris, John Libbey Eurotext, p. 45-65.

BATT-MOILLO, A. et A. JOURDAIN (dir.) (2005). Le suicide et sa prévention, Paris, Édition ENSP.

BAYET, A. (1922). Le suicide et la morale, Paris, Libraire Félix ALCAN.

BECK, U. (2001). La société du risque, Paris, Flammarion.

BERGER, P. et T. LUCKMANN (1966). La construction sociale de la réalité, Paris, Armand Colin.

BOURDELAIS, P. (dir.) (2001). Les hygiénistes, enjeux, modèles et pratiques, Paris, Belin.

BURTON-JEANGROS, C. (2004). Cultures familiales du risque, Paris, Antropos, coll. «Sociologique».

CAMPEON, A. (2005). «La construction politique contemporaine d'un problème de santé publique », dans BATT-MOILLO, A. et A. JOURDAIN (dir.), Le suicide et sa prévention, Paris, Éditions ENSP, p. 65-79.

CHAGNON, F. et B.L. MISHARA (dir.) (2004). Évaluation de programmes en prévention du suicide, Québec, Presses de l'Université du Québec.

DAUZAT, P.-E. (1998). Le suicide du Christ, Paris, Presses universitaires de France.

DAVIDSON, F. et A. PHILIPPE (1986). Suicide et tentatives de suicide aujourd'hui, Paris, Éditions INSERM et DOIN.

DEBOUT, M. (2002). La France du suicide, Paris, Éditions Stock.

DECHOZ, J. (1994). Les rites du suicide, mémoire, Université d'Angers.

DE LEO, D. (2005). «Bilan des politiques de prévention: pourquoi si peu d'avancées?", dans BATT-MOILLO, A. et A. JOURDAIN (dir.), Le suicide et sa prévention, Paris, Édition ENSP, p. 327-258.

DOZON, J.-P. et D. FASSIN (dir.) (2001). Critique de la santé publique. Une approche anthropologique, Paris, Éditions Balland, coll. «Voix et regards».
DURKHEIM, É. (1897). Le suicide, Paris, Presses universitaires de France.

ERNER, G. (2006). La société des victimes, Paris, La Découverte.

FACY, F. et M. DEBOUT (2006). Acteurs et chercheurs en suicidologie, Paris, Éditions EDK.

FARHI, R. (2003). Le suicide en France: opinion des Français, Paris, UNPS et L'Harmattan.

FOUCAULT, M. (1976). La volonté de savoir, Paris, Gallimard.

GUILLON, C. et Y. LE BONNIEC (1982). Suicide mode d'emploi. Histoire technique et actualité, Paris, Éditions Alain Moreau.

GUIRLINGER, L. (2000). Le suicide et la mort libre, Nantes, Éditions Pleins Feux.

HARDY, P. (2000). «Peut-on évaluer le rôle des services d'assistance téléphonique dans la crise suicidaire? ", dans FFP, La crise suicidaire, reconnaître et prendre en charge, Paris, John Libbey Eurotext, p. 311-325.

HAWTON, K. et K. VAN HEERINGEN (dir.) (2000). The International Handbook of Suicide and Attempted Suicide, Chichester, Wiley \& Sons Ltd.

MACQUET, C. et D. VRANCKEN (2006). Le travail sur soi, Paris, Belin.

MISHARA, B.L., F. CHAGNON, M. DAIGLE, B. BALAN, S. RAYMOND, I. MARCOUX, C. BARDON et J.K. CAMPBELL (2005). Do Helplines Help Suicidal Callers? Results of the AAS-Hopeline Silent Monitoring Evaluation Study. Document présenté à la Canadian Association for Suicide Prevention Conference, Ottawa, Canada.

MINOIS, G. (1995). Histoire du suicide, Paris, Fayard.

LUSTMAN, M. (2008). Du suicide comme construction sociale, thèse, Université Paris-Descartes.

SILVERMAN, M. (2005). "Suicide prevention strategies: A systematic review ", Jama, vol. $294, \mathrm{n}^{\circ} 16,26$ octobre

TUDOR, K. (1996). Mental Health Promotion. Paradigms and Practice, Londres, Routledge.

\section{Notes}

1. L'article est fondé sur notre thèse de sociologie: Du suicide comme construction sociale (2008).

2. Étude en cours de réalisation: Comparaison des pratiques de la téléphonie sociale entre la France et le Québec. 